\title{
Ectopic left cervical thymic tissue in pediatric age group
}

\begin{abstract}
Objective: We report a case of ectopic cervical thymic tissue in a healthy 5years11 months old girl.

Method: Case report and review of the English-language literature (using PubMed, Ovid, and Proquest databases).

Results: Case of ectopic cervical thymic tissue occurred in a preschool-aged girl to be reported in the English-language literature.

Conclusion: This case raises the alerts of ectopic cervical thymic tissue to be considered in the differential diagnosis of children younger than 10years of age with a painless neck mass. When previously healthy child manifests with a sudden onset of the asymptomatic neck mass, keep in your mind that there is a great likelihood of ectopic thymus tissue in the neck.
\end{abstract}

Keywords: thymus gland, ectopic tissue, pediatric neck mass, fibrocollagenous tissue, ectopic thymus cyst
Volume 6 Issue 5 - 2017

\author{
Misheal Madni,' Laila Salah Seada, ${ }^{2}$ Adel \\ Ahmed Alfayez, ${ }^{3}$ Amjad Tallaa Atallaa, ${ }^{3}$ \\ Meshael Salem Alshammari, ${ }^{3}$ Salwa Menwer \\ Alanazi $^{3}$ \\ 'Consultant and chief of pediatrics surgery Department, King \\ Khalid Hospital, Saudi Arabia \\ ${ }^{2}$ Consultant Histopathology, King Khalid Hospital, Saudi Arabia \\ ${ }^{3}$ University of Hail, Saudi Arabia
}

Correspondence: Adel Ahmed Alfayez, University of Hail, Saudi Arabia, Email adelaalfayez@gmail.com

Received: April 22, 2017| Published: May 03, 2017

\section{Summary}

Ectopic thymus tissue in the neck remains infrequently described in medical literature. This paper presents the case of a young girl, who presenting with a soft, fluctuating bulk mass on the left side of her neck. The patient underwent complete excision of the lesion and histological inspection showed an ectopic thymic tissue cyst. Ectopic thymic soft tissue may remain an uncommon finding, but it must be involved in the differential diagnosis of neck masses, particularly in children. This case report is complemented by a short review of the relative literature.

\section{Background}

The thymus is a major lymphoid tissue in the embryonic stage and early childhood. It mainly appears early in fetal life as well as plays a critical role in the development of cell-mediated immunity. Ectopic cervical thymus is not often reported, for the reason that thymic remnants frequently persist as a symptomatic and unrecognized by clinicians. Most of these lesions arise as a result of migration defects throughout thymic embryogenesis. Since 1901, about 90 cases of aberrant cervical solid and cystic thymic lesions have been reported in the literature and were recognized at either surgery or autopsy. ${ }^{1-3}$ Approximately two-thirds of all reported cases were identified in children younger than 10years. ${ }^{4}$ Ectopic thymic tissue in the neck is unusual in patients older than 20years.

\section{Case presentation}

A 5-year and 11months old girl, previously healthy, presented in our emergency (ER) department with a left cervical swelling since 4 months. It has gradually increased in size and was not associated with fever or weight loss, as the mother mentioned. On examination, the child looked well, with an average body weight, neither pale nor jaundiced. Local examination revealed a left anterior fixed, nonmobile, soft cervical neck mass. No tenderness on palpation. No discharge was coming out and no redness or hotness. After that the patient was admitted in our hospital and investigations were done for her, she underwent surgical excisional biopsy and stayed 4days in the Hospital. The histopathology report came to be "a thymic cyst with multiple foci of ectopic thymus gland tissue"(Figures 1-9).

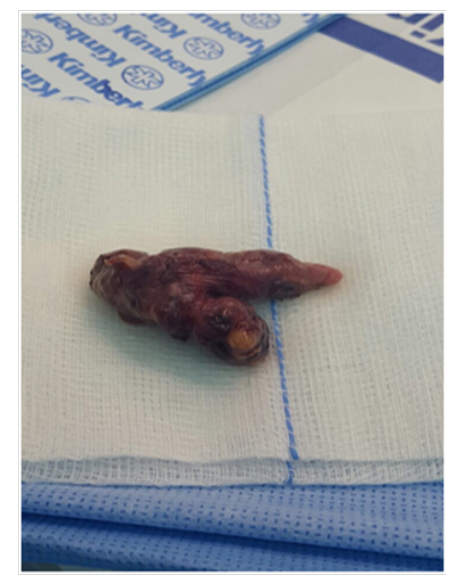

Figure I Gross appearance of the mass.

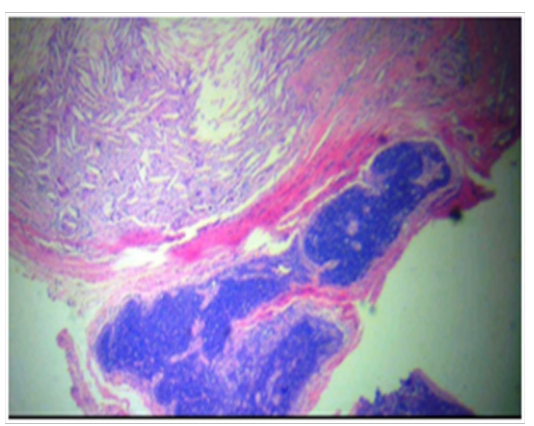

Figure 2 Thymic cyst showing cholesterol crystals. 


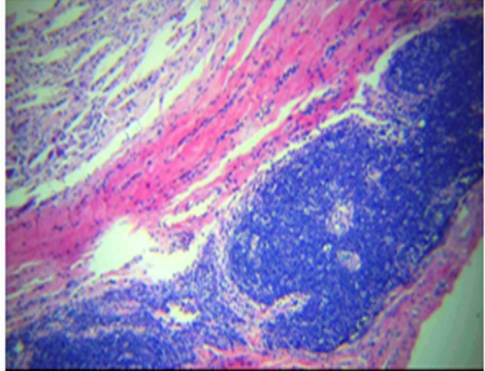

Figure 3 Ectopicthymic tissue.H\&Ex200.

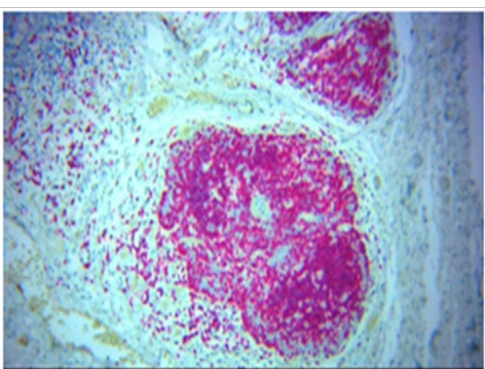

Figure 4 Thymic tissue: CD3 positive T lymphocytes.

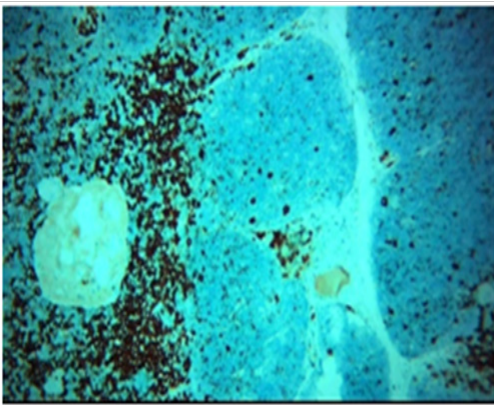

Figure $5 \mathrm{CD} 20$ negative in thymic tissue. Tissue APAAP, red chromogenx200Peroxidase, DAB chromogen $\times 200$.

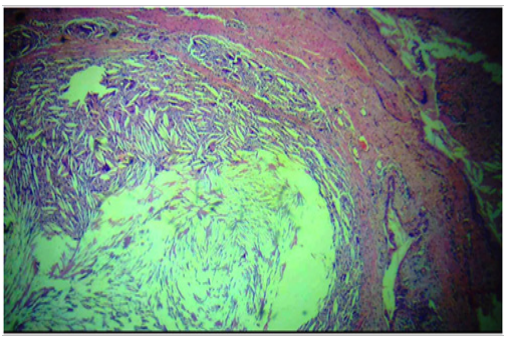

Figure 6 Thymus cyst cholesterol crystal clefts H\&E x400



Figure 7 Chest and neck $X$-ray figure out and confirm that "there is no mediastinal extension of the mass".

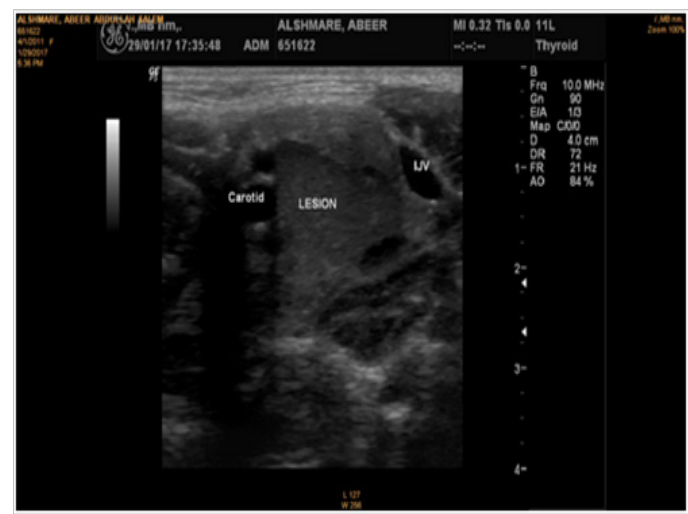

Figures 8 Ultrasound of the neck shows large heterogeneous lesion.

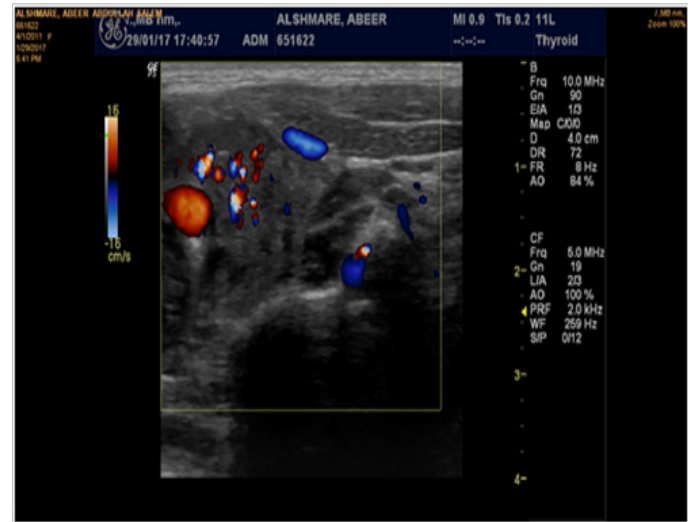

Figures 9 Ultrasound of the neck shows large heterogeneous lesion which is seen in the anterior triangle of a left cervical region, between the internal jugular vein and the carotid artery, displacing the carotid anteromedially and the jugular posteriorly; it measured about $3 \times 1.5 \times 1.5 \mathrm{~cm}$, it shows heterogeneous hypervascular texture with focal calcification. For clinical correlation and further investigation considering Carotid Space masses (paraganglioma, chwannoma, others).

\section{Surgical pathology specimen - profile}

\section{Gross description}

One piece of an irregular nodular, firm soft tissue bit measuring $4 \times 2 \times 1 \mathrm{~cm}$. Cut section showed multiple small grayish-white nodules largest measurements. $1 \times 0.5 \mathrm{~cm}$ and smallest $0.3 \times 0.3 \mathrm{~cm}$, multiple bits, 5blocks, all embedded.

\section{Microscopic picture}

In multiple bits of the tissue received, multiple cystic spaces are seen, some are rounded others are irregular. Cysts lining ranged from the cubical stratified epithelium. Other cysts form a fibrous capsule. Cysts are filled with huge numbers of multinucleated gains cells and large numbers of cholesterol crystals. Outskirts show multiple foci of thymus gland tissue with a well-formed cortex of packed, monotonous lymphoid T-cells, with tangible body macrophages. Medulla show less packed lymphoid cells. Epithelial whorls of Hassall's corpuscles are evident. Fibrocollagenous tissue surrounding the cysts show congested capillaries. No evidence of Malignancy.

\section{Differential diagnosis}

The differential diagnosis of cervical thymic cyst is broad and includes thyroglossal duct cyst lymphovascular malformations, branchial cleft cyst, and laryngocele, as well as benign tumors (dermoid 
cysts, epidermoid cysts) and malignant tumors (lymphoproliferative, soft tissue sarcoma and other metastatic lesions). ${ }^{5}$

\section{Outcome and follow up}

The operation was done under general Anesthesia through left cervical incisional approach. The pre-operative and postoperative diagnosis was "left cervical mass-possible schwannoma respectively". Excisional biopsy was done for her and the large tumor situated between left internal carotid artery \& internal jugular vein. It was attached to nerve sheath. Left sternomastoid Muscle retracted laterally and Tumor of variable consistency arising deep to sternomastoid Muscle between internal-carotid Artery\& internaljugular Vein attached to nerve sheath. Dissection of a carotid sheath and surrounding tissue was done and the complete mass removed. Repair of nerve was done with 6 Prolene. Her condition improved postoperatively and subsequently, she was discharged from our hospital. Currently, she's doing well and had been followed up in pediatrics surgical clinic.

\section{Discussion}

Cervical thymus cysts (CTC) are epithelial-lined cysts that most commonly present during the first decade of life, with a slight preponderance towards males. ${ }^{6}$ Incidences of ectopic thymic tissue in the neck have been reported in various conditions, despite their diversity, there are only a few studies focusing on patients without any thymic disease. ${ }^{7}$ CTCs can be either congenital or acquired and are most commonly found in the left sided neck, anterior to the SCM, with approximately 50\% showing extension into the superior mediastinum. ${ }^{8}$ Since CTCs represent less than $1 \%$ of cystic cervical masses, they are often overlooked in the broad differential diagnosis of children presenting with painless neck masses. It is important, however, for physicians to be able to efficiently and accurately diagnose CTCs since they can usually be surgically excised with little risk of postoperative recurrence. ${ }^{9}$ The thymus gland is mainly originated from the third and fourth pharyngeal pouches in association with the inferior parathyroid glands. Right and left portions of the thymic primordium descend down the neck during the sixth to eight weeks of gestational life and fuse to form the gland. ${ }^{9}$ There are three mechanisms for pathogenesis of ectopic thymus in the neck: aberrant descent, sequestration of thymic tissue and failure of involution. ${ }^{10}$ Most patients with thymic cysts complain of a slowly enlarging, a symptomatic cervical mass. Symptomatic cases of cervical thymic cysts are so rare that this diagnosis is not common preoperatively. In patients with myasthenia gravis, hyperplasia of thymic tissue occurs in their thymus gland and ectopic thymic tissue as well. As a result, the probability of finding ectopic thymic tissue in such a patient is higher than in patients without any thymic disease. ${ }^{7}$ Diagnosis by fine needle aspiration was appropriately described. ${ }^{11}$ There has been no reported recurrence and long-term prognosis is excellent.

\section{Learning points}

i. Ectopic Thymus cyst must be considered in differential diagnosis of a young child with a cervical mass.

ii. Once diagnosed, surgery is the definitive treatment. The utility of fine needle aspiration is gaining wider acceptance in the diagnostic evaluation of neck masses.

iii. The prognosis after removal of ectopic cervical thymus is excellent and no cases of recurrence have been reported.

iv. Such anomalies are rarely diagnosed pre-operatively and can be easily confused with other neck lesions.

v. Mediastinal thymus should be confirmed to prevent the risk of a total thymectomy.

\section{Acknowledgements}

None.

\section{Conflict of interest}

The author declares no conflict of interest.

\section{References}

1. Nguyen Q, de Tar M, Wells W, et al. Cervical thymic cyst: case reports and review of the literature. Laryngoscope. 1996;106:247-252.

2. Loney DA, Bauman NM. Ectopic cervical thymic masses in infants:a case report and review of the literature. Int J Pediatr Otorhinolaryngol. 1998;43(1):77-84.

3. Millman B, Pransky S, Castillo J, et al. Cervical thymicanomalies. Int J Pediatr Otorhinolaryngol. 1999;47(1):29-39.

4. Bieger RC, McAdams AJ. Thymic cysts. Arch Pathol. 1996;28:535-541.

5. Statham MM, Mehta D, Willging JP. Cervical thymic remnants in children. Int J Pediatr Otorhinolaryngol. 2008;72(12):1807-1813.

6. Bruvère PJ, Moreau P, Ghave B. Congenital thymic cyst. JBR-BTR. 2007;90(3):178-179.

7. Tabatabaie SA, Hashemi SM, Sanei B, et al. The frequency of ectopic thymic tissue in the necks of patients without any thymic disease. Med Sci Monit. 2007;13(6):CR283-285.

8. Hsieh YY, Hsueh S, Hsueh C, et al. Pathological analysis of congenital cervical cysts in children: 20years of experience at chang gung memorial hospital. Chang Gung Med J. 2003;26(2):107-113.

9. Sturm-O'Brien AK, Salazar JD, Byrd RH, et al. Cervical Thymic anomalies-the Texas Children's Hospital experience. Laryngoscope. 2009;119(10):1988-1993.

10. Tovi F, Mares AJ. The aberrant cervical thymus. Embryology, Pathology,and clinical implications. Am J Surg. 1978;136(5):631-637.

11. Tunkel DE, Erozan YS, Weir EG. Ectopic cervical thymic tissue diagnosis by fine needle aspiration. Arch Pathol Lab. 2001;125(2):278-281. 